\title{
Introduction: Des castors à la terre : Construire sur les débats passés pour défaire l'enchevêtrement contemporain des territoires de chasse familiaux des Algonquiens
}

\author{
Mélanie Chaplier McGill University/Université Catholique de Louvain \\ Colin Scott McGill University \\ Translated from the original by Aurélie Maire
}

Résumé : En 1986, Anthropologica publiait un numéro spécial sur les territoires de chasse familiaux Algonquiens comportant diverses recherches ethnographiques qui renversaient, ancraient ou recadraient les travaux antérieurs portant sur les origines ou décrivant le communisme primitif des systèmes de propriété foncière Algonquiens. Le numéro présentait des recherches développées dans le cadre de la montée de revendications politiques et juridiques Autochtones pour le maintien de leur mode vie et de leur gouvernance territoriale alors que se multipliaient les interventions économiques et étatiques sur leurs terres. Dans cette Introduction au numéro thématique, nous présentons un aperçu historique ainsi qu'un nouveau cadre conceptuel pour analyser la territorialité et la gouvernance autochtones, informés par la manière dont les Algonquiens continuent de répondre aux défis auxquels ils font face depuis trente ans. Nous décrivons l'évolution de la gouvernance et des façons de vivre des Algonquiens parmi les initiatives d'extraction et d'exploitation des ressources sur leurs terres, ainsi que certaines transformations importantes au sein des socialités Algonquiennes.

Abstract: In 1986, Anthropologica published a special issue on Algonquian Family Hunting Territories (FHT) with diverse ethnographic research that overturned, grounded and reframed the earlier literature on the origins and the private-primitive communism property descriptions of Algonquian land tenure systems. The issue presented research developed with. for and in the emerging northern Indigenous political and legal struggles to continue to live on and govern their lands in the midst of rapid economic and state interventions. In this Introduction to the special issue, we provide a historical overview as well as a renewed framework for the analysis of Indigenous territoriality and governance which has been informed by the ways Algonquian peoples have continued to respond to the challenges they faced in the last thirty years. We describe the evolution of the Algonquian lives on the land and governance in the midst of resource exploitation and extraction, as well as important shifts within continually emerging Algonquian socialities.
$\mathrm{I}^{1}$ l y a trente ans, la revue Anthropologica publiait un numéro spécial sur les territoires de chasse familiaux des Algonquiens ${ }^{1}$ (TCF), un régime foncier autochtone identifié pour la première fois dans la littérature anthropologique par Speck en 1915 et l'objet d'intenses débats durant le $\mathrm{XX}^{\mathrm{e}}$ siècle. Comme question centrale, les éditeurs invités de ce numéro spécial se sont demandés : " qui est propriétaire des castors ? ", un choix de mots ciblant clairement dans ce débat le commerce de la fourrure, bien que le castor était aussi, bien sûr, et l'est toujours, une source de nourriture importante. À l'époque, et selon les publications récentes à la fois en ethnohistoire (Morantz 1978 et en anthropologie (Tanner 1971 ; Feit 1982), cette publication a contribué à changer le regard du « débat classique » sur les origines de cette institution - vue comme " une question restrictive et simple» - pour investiguer « une variété de questions portant sur l'exploitation actuelle des territoires de chasse en tant que système foncier dans des cas ethnographiques et historiques spécifiques " (Tanner 1986 : 22). De différentes manières, cette publication de 1986 fut un moment décisif entrainant l'émergence de différents types de recherches anthropologiques qui inclurent progressivement divers aspects de gestions de la terre des Peuples Algonquiens (particulièrement les Cris). Avec ce nouveau numéro spécial, nos efforts suivent manifestement cette veine en prenant en compte la signification, parfois déstabilisante, des changements qui se produisent sur les terres des Algonquiens à l'aube du XXIe siècle. Si le « renouveau » de ce débat peut être convenablement discuté, notre tentative vise plutôt à intégrer les derniers changements survenus sur les terres algonquiennes, mais aussi les perspectives anthropologiques les plus récentes, que de rouvrir de vieilles questions $^{2}$. En nous basant sur le travail de nos prédécesseurs, nous décrirons les multiples manières selon lesquelles les TCF sont devenus un enjeu politique majeur, intégrés dans un large ensemble d'activités - la 
négociation des revendications territoriales, l'exploitation des ressources, la redéfinition des lois coutumières et le développement socio-économique - tout en restant au centre des pratiques cynégétiques quotidiennes. Ce faisant, ce numéro décrira le rôle des TCF en tant qu'institution au milieu de l'exploitation de ressources et d'importants changements sociétaux, tout en s'interrogeant sur les formes contemporaines de ces territoires et leur meilleure intégration au sein des politiques de développement régional.

\section{Sur les limites de l'imagination anthropologique}

Parmi les thèmes variés qui ont marqué l'anthropologie canadienne, le débat autour des territoires de chasse familiaux des Algonquiens a occupé une place prépondérante. Depuis la « découverte » de Speck $(1915,1923$, 1927) et sa description du territoire de chasse familial comme « un groupe de parents composé de personnes unies par le sang ou le mariage, ayant le droit de chasser, de trapper et de pêcher dans une certaine région reçue en héritage et délimitée par quelques rivières, lacs ou autres frontières naturelles " (Speck 1915 : 290), beaucoup a été écrit au sujet de ce régime foncier. Depuis des décennies, des camps se sont vivement opposés avec des idées très arrêtées (pour un compte rendu détaillé, voir la contribution de Morantz dans ce numéro). Il est bien connu que ce débat a dépassé la territorialité des Algonquiens pour les questions théoriques plus larges de nos chers anthropologues marxistes et boasiens, à savoir l'évolution de la sociabilité humaine et l'histoire de l'idée de propriété. Alors que nous souhaitons proposer ici une brève rétrospective, notre intérêt n'est pas de détailler chaque position et acteur abondamment décrits ailleurs (Pulla 2006, 2011 ; Tanner 1986 ; Morantz, dans ce numéro). Toutefois, il y a un intérêt à décortiquer le rôle que joue l'anthropologie en tant que discipline pour la compréhension et la description des territorialités autochtones. Depuis la perspective évolutionniste contestée au début du XXe siècle, au travers de concepts comme l'acculturation et l'assimilation, et jusqu'aux notions contemporaines d'hybridité et d'enchevêtrement, se déroule une captivante saga intellectuelle. Nos efforts à décrire cette chronique offrent ainsi un point de vue critique fertile sur notre discipline.

Nous suggérons une triple caractérisation des critiques étroitement imbriquées et récurrentes qui ont visé le débat original. Tout d'abord, un criticisme important a condamné l'attention exagérée accordée aux origines des FHT et à leur relation aux théories évolutionnistes. En effet, jusqu'aux années 1970, « les sociétés nordiques étaient avant tout considérées comme un apport de preuves pour ou contre des hypothèses spécifiques d'organisation sociale » (Cruikshank 1993 : 135). Alors que les scientifiques occidentaux étaient profondément imprégnés de cette perspective évolutionniste, la fascination de l'anthropologie pour la question des origines ${ }^{3}$ de la société a emprisonné les institutions et les pratiques territoriales du Peuple Algonquien - plus variées et nuancées que ce qui avait été pris en compte - dans un portrait ethnocentrique, simplifié et parfois romantique. Par conséquent, l'anthropologie à ses débuts - et la société coloniale dans son ensemble - a été incapable de bien comprendre et de décrire les relations alternatives à la terre. Finalement, l'attention accordée à « la question de l'autochtonie est, dans les faits, un débat sur les origines d'une institution que nous ne comprenons pas » (Tanner $1986: 23)^{4}$. Il apparaît cependant plus facile de juger des anthropologues passés selon les critères actuels, mais personne ne peut nier que la discipline, pratiquée dans ce contexte spécifique, n'avait ni les outils, ni les concepts ou l'imagination pour décrire les régimes fonciers basés sur des principes ontologiques différents ${ }^{5}$.

La seconde critique importante, formulée par plusieurs auteurs dans le numéro spécial de 1986 (Bishop, Tanner, Morantz), cite la tendance problématique de généraliser à partir d'un cas local particulier à la famille sociolinguistique des Algonquiens en général. Comme le débat s'est endurci, les auteurs ont parfois extrapolé certaines données pour étayer leur propos au groupe entier des Algonquiens, avec parfois peu de considération pour les différences importantes dans les contextes sociaux, culturels et écologiques de chaque communauté6. On pourrait argumenter le fait que la nature adverse de ce débat a ouvert l'espace à la surinterprétation et à une vision restreinte. Comme un signe, les TCF ont été " découverts " dans le monde autochtone canadien et bien au-delà.

Troisièmement et finalement, nous pouvons pointer les limites de la nature dichotomique de ce débat. La plupart des acteurs du débat classique ont développé une perspective « soit-ou » ne permettant pas encore la description des zones grises. Par exemple, les TCF des Algonquiens sont soit des Autochtones ou des émergents du commerce de la fourrure, soit une forme de propriété communale ou de propriété privée. Cette nature dichotomique a de profondes conséquences pour notre compréhension de la transformation socioculturelle que confrontent ces groupes. Comme l'a noté Tanner, les anthropologues tendent à présenter les peuples non occidentaux selon deux positions opposées : "Soit ils sont vus comme vivant un mode de vie basé sur des 
conditions locales et une culture distincte ou, dans l'autre extrême, ils sont portraiturés comme des minorités dominées, déterminées ou absorbées par une société majoritaire coloniale ou industrielle » (Tanner 1983 : 312). L'incapacité anthropologique à dépasser ces positions de "soit-ou » et de penser en termes d'hybridité et d'enchevêtrements complexes (en d'autres termes, dans une perspective de « et-et » ou « ni-ni ») a généré des conclusions discutables. Lors de l'évolution récente de ce débat, le travail de Leacok a nourri l'émergence de la formulation de théories en termes d'acculturation et d'assimilation. Dans leur remarquable article sur " les trappes et les trappeurs ", Murphy et Steward (1956) ont insisté sur l'assimilation en cours et inévitable des sociétés égalitaires (band societies) vers des économies industrielles ${ }^{7}$. Cette perspective qui dénonce la déliquescence des modes de vie autochtones a été largement utilisée dans les travaux scolaires avec les Peuples autochtones du Canada jusqu'à la fin des années soixante. Plus tard, elle a été combattue par une nouvelle génération d'anthropologues et de leaders autochtones (Cruikshank 1993 : 135-136).

Ces trois caractéristiques entremêlées du débat original entourant les TCF des Algonquiens identifient toutes un manque de fluidité et de dynamique dans l'étude des droits de propriété autochtones. Sont en jeu les limites des anthropologues du passé à comprendre la complexité sociale qui a émergé de la rencontre coloniale au Canada. La disparition ou l'assimilation des pratiques de chasse et des visions du monde autochtones n'ayant pas été une conséquence historique de la colonisation, de nouvelles données ethnographiques et perspectives théoriques ont contribué à changer la manière de comprendre la résilience des TCS en tant qu'institution centrale.

\section{L'ethnographie des territoires de chasse familiaux contemporains}

Comme nous l'avons mentionné plus haut, une génération d'anthropologues nouvellement formés a pris place durant le dernier tiers du vingtième siècle et renouvelé le corpus des données ethnographiques disponibles sur les TCF, avec un intérêt plus marqué pour les Cris de la Baie-James. Une série d'évènements et de raisons ainsi qu'une bonne dose de coïncidences - explique cet intérêt pour les Cris. Nous mettrons ici en lumière trois facteurs.

Le premier est la création du Projet McGill-Cri (McGill-Cree Project) de Norman Chance, en 1964, qui tombe à point nommé ${ }^{8}$. Eu égard au nouvel intérêt pour le développement social des Peuples autochtones issu de la commission Hawthorne-Tremblay, l'équipe de chercheurs $^{9}$ du Projet McGill-Cri a examiné les changements économiques, sociaux et politiques en cours dans les communautés cries du Sud. Partant initialement de la perspective d'une acculturation, le projet s'est attaché, en premier lieu, à l'industrialisation des Cris (Chance 1968) ${ }^{10}$, non pas à la territorialité ou aux pratiques de chasse. En tant que tel, le Projet McGill-Cri n'a pas généré un regain d'intérêt pour les TCF, mais a créé les conditions pour que cela émerge. En effet, une nouvelle génération d'anthropologues a été formée dans le cadre de ce projet - dont certains d'entre eux se sont ensuite penchés sur la territorialité - et le projet a créé l'impulsion pour les partenariats entre les chercheurs de l'université McGill et les Cris ; des collaborations qui sont devenues centrales au cours des décennies suivantes.

Cela nous amène à la seconde raison plus significative quant à l'attention anthropologique soutenue accordée aux Cris : le Projet de Baie-James. Là encore, le projet n'a pas déclenché un intérêt scolaire pour les TCF - Feit, Tanner et Preston avaient tous mené leur enquête de terrain avant l'annonce du projet - mais a apporté pertinence et durabilité à leur recherche. L'ampleur de ce projet de développement des ressources ${ }^{11}$, la soudaineté de son annonce et la réponse rapidement donnée aux Cris (et aux Inuit) a effectivement généré un large soutien universitaire ${ }^{12}$. De plus, les TCF cris sont devenus une affaire d'intérêt public. Les experts, aux côtés des chasseurs cris, ont témoigné de leur existence au tribunal et la curiosité des médias pour la relation des Cris avec leurs terres s'est accrue (voir Richardson 1975). Par ailleurs, la signature de la Convention de la Baie-James et du Nord québécois (CBJNQ) en 1975 et ses conséquences prévues ont justifié une attention soutenue pour le futur des Cris. Cette entente et son application ont également redéfini le rôle des anthropologues (comme experts et avocats) puisqu'ils ont suivi les Cris au travers des transformations socio-économiques complexes et effrénées, mais aussi au travers de leur lutte à pouvoir s'exprimer sur la gestion et le développement de leurs terres.

Une troisième raison crédible pour la prédominance de l'étude des TCF des Cris est leur résilience tout à fait remarquable. Il y a pour cela deux explications. Tout d'abord, en raison de leur isolement relatif, les Cris ont subi moins de pression et d'empiètement sur leurs terres que les groupes d'Algonquiens plus au Sud, pendant le commerce de la fourrure et l'histoire du début de l'extraction industrielle des ressources. Par conséquent, leurs activités cynégétiques étaient encore florissantes au début des années 1970 (avec quelques variations 
d'une communauté à l'autre). Ensuite, les TCF des Cris étaient fortement reconnaissables parce qu'ils avaient été, durant des décennies, progressivement intégrés dans la structure administrative de la gestion des ressources. Cela a été présenté en détail par Toby Morantz (2002) et discuté plus récemment par Scott et Morrison (2004, 2005), Harvey Feit (2009a) ou Susan Preston (2011). Plusieurs vagues d'intervention externes - la préservation des castors, les territoires de chasse enregistrés et la CBJNQ - ont participé à un lent changement des TCF vers un régime foncier plus institutionnalisé et graduellement cartographié plus strictement $^{13}$. Ce processus était sûrement non exempt de dissonance fonctionnelle, car l'on pouvait - et peut toujours - observer un fossé entre ces cartes d'apparence formelle et la fluidité et la nature négociable des pratiques quotidiennes de la cohabitation sur le territoire. Toutefois, cette formalisation a participé à l'endurance et à la haute visibilité de cette forme de régime foncier.

Encouragés à plusieurs niveaux par ce contexte, quatre universitaires ont été à l'avant-garde de la recherche sur les pratiques cynégétiques des Cris au début des années 1970 : Richard Preston, Adrian Tanner, Harvey Feit et Colin Scott ${ }^{14}$. Tandis que les trois premiers ont mené leurs enquêtes de terrain à la fin des années 1960 - respectivement, à Waskaganish, à Mistissini et à Waswanipi -, Scott a débuté sa recherche à Wemindji, approximativement une décennie plus tard. Comme mentionné plus haut, leurs travaux étaient basés sur l'affirmation que les TCF cris n'étaient pas une réponse au commerce de la fourrure (ou à tout autre élément externe), mais " un moyen critique de la reconstitution des larges relations sociales et des significations symboliques de base des chasseurs cris " aussi bien qu' « un moyen essentiel de l'élaboration de changements locaux engendrés par les liens grandissants entre les nations états et les marchés " (Feit 1991a : 224). Leurs données ethnographiques, alors qu'elles démontraient la continuité et le chevauchement géographique avec la description de Speck, ont aussi révélé que le système était en train de devenir plus rigide et formalisé (Tanner 1979 ; aussi observé dans Craik et Casgrain 1986). Leur analyse a permis une "nouvelle reconnaissance de la résilience des relations sociales de la chasse communale qui, dans de nombreux cas, a résisté à des siècles de participation à l'économie capitaliste et aux classes politiques » (Scott 1986 : 164). Grâce à leurs recherches, une définition affinée et plus contemporaine des TCF a émergé. Selon Harvey Feit :
Les éléments clés des systèmes de territoire de chasse sont : un intérêt commun et inaliénable dans l'utilisation et la protection de toutes les ressources du territoire ; l'existence d'un ensemble d'intendants relativement stable et limité dont les savoirs, les liens spirituels existants et les lopins de terre sont à la base de leur autorité au-delà de l'utilisation intensive de ces territoires et des ressources par les membres de la communauté ; les attentes de la communauté, la sanction et l'encouragement des leaders pour exercer l'autorité au regard de la protection de la communauté et des besoins des familles, la continuité intergénérationnelle et les besoins de tous d'accéder à la terre. (1991 : 230)

Depuis plusieurs décennies et toujours actuellement, le travail de ces quatre chercheurs ${ }^{15}$ a produit une littérature importante et a expliqué plusieurs sujets clés. D'abord, ils ont développé une perspective considérablement plus nuancée sur le débat concernant la propriété, discutant les nombreuses possibilités du système de même que le vocabulaire en jeu - propriété privée, propriété collective, l'intendance et l'usufruit. Dans le numéro spécial d'Anthropologica, en 1986, ils ont exprimé leurs critiques au regard du manque de précision du débat original et ont approuvé le rejet de l'idée que les TCF soient une propriété privée ${ }^{16}$. Alors que Tanner utilise le terme " usufruit » pour décrire ce régime foncier, il souligne que la terre en soi «n'est pas propriété » et que les TCF sont plus efficacement décrits comme « des unités de gestion» (1986). C'est une position soutenue par plusieurs auteurs selon lesquels les « Cris ne voient pas la terre comme un état réel » (Berkes 1986, 150) et la relation de propriété des intendants à leurs territoires de chasse "n'est pas celle de la propriété par les marchés standards » (Feit 1991a : 229 ; voir aussi Nadasdy 2002). Deux ans plus tard, Scott résume leurs arguments :

Pour parler de la propriété crie - même de propriété collective -, il faudrait faire abstraction de la dynamique essentielle du système. Les droits coutumiers dans le territoire, les ressources de subsistance et les productions peuvent être spécifiés, mais cela se rapporte aux relations politiques et techniques de gestion et de partage des ressources - ressources dans lesquelles personne, dans la dernière analyse, ne détient des droits exclusifs ou absolus. (Scott 1997 [1988] : 40)

Dans leur effort à décrire les pratiques territoriales des Cris - et c'est le second - ces auteurs ont également 
mis en lumière le rôle du chef de chasse, aussi appelé le maître-trappeur ou nituuhuu uиchimaau. L'attention accordée aux divers aspects de ce rôle central dans la société crie a été une évolution majeure dans le débat (qui n'était plus du tout un débat). En décrivant la « forte éthique égalitaire ", sur laquelle s'appuie l'autorité du maitre-trappeur, ils ont enrichi notre compréhension des TCF :

En reconnaissant et en favorisant des relations correctes envers " les personnes ", le leader profite de l'autorité pour « décoder ». Vis-à-vis des populations animales, cette responsabilité inclut la connaissance du nombre des animaux qui devraient être collectés par le groupe, du lieu et du moment de cette collecte, pour maximiser le bénéfice social, tout en maintenant des conditions écologiques optimales. Vis-à-vis des êtres humains, cette responsabilité implique le partage généreux des opportunités de chasse. (Scott 1997 : 38-39)

Troisièmement, en associant les aspects écologiques, sociaux et symboliques des TCF, ils ont rejoint ce qui manquait dans le débat classique, c'est-à-dire une compréhension plus holistique. Leurs ethnographies comportent des descriptions de la construction sociale complexe de la chasse crie, incluant des aspects qui ne sont pas «mis en mots " puisqu'ils sont « davantage incarnés par des attitudes et des actions que des pensées conscientes ou des réflexions »(Preston $1986: 15)$. Ils décrivent particulièrement la nature spirituelle des pratiques de chasse du quotidien - ce que Tanner (1979 : 108) appelle la « religion de la chasse ${ }^{17}$ " (hunting religion) - comme une importante caractéristique des TCF. En effet, " on dit de l'intendant qu'il a des liens très étroits avec les esprits de la terre qu'il possède » (Feit 1991a : 229). Ces perspectives sur la nature religieuse de la chasse ont été tardivement formulées en tant qu'étude de "l'ontologie ", selon des débats renouvelés de la discipline (Descola 2005 ; Ingold 2000 ; Scott 2013). Il avait été ainsi souligné que dans le monde de « l'ontologie relationnelle » (Poirier 2017 ; Scott 2017), les animaux se donnent volontairement (mais sans) au chasseur respectueux qui, par conséquent, manifeste l'attitude appropriée envers ces personnes humaines à part. À cet égard, les idées de respect et de partage inhérentes aux TCF sont perceptibles dans de nombreuses interactions sociales; elles existent dans les pratiques cynégétiques ou dans les interactions avec la société colonisatrice. En effet, alors que l'ensemble de la société crie relevait des défis majeurs face à l'exécution de la CBJNQ, une importante part du travail des chercheurs était consacrée à la description du rôle joué par les TCF dans le contexte des changements politiques et socio-économiques. Opposées aux théories d'acculturation du passé, ces analyses mettent en lumière la façon dont les TCF étaient un lieu de résistance et d'affirmation de l'identité culturelle crie, même sous une pression externe grandissante. Tout en reconnaissant la transformation de l'économie crie - l'augmentation des transferts d'argent liquide, l'explosion des opportunités d'emploi à temps plein et la marchandisation des ressources - les chercheurs ont largement démontré la résilience, par l'intermédiaire des TCF, des éthiques de la chasse susmentionnées. Scott (1986 : 170), par exemple, observe : « il n'apparait pas que la simple implication avec les économies capitalistes au niveau de la marchandisation des produits soit une condition suffisante pour l'érosion des régimes fonciers autochtones ou pour la transformation fondamentale des relations collectives fécondes ". Assurément, au fil du temps, les tensions ayant pour enjeux l'accès à la terre et aux ressources sont à la hausse et les incitations à la privatisation deviennent manifestes. En même temps, " il y a des moyens de résistance potentiellement efficaces aux efforts occasionnels d'un petit nombre d'intendants, dans le but d'améliorer la monétarisation des invitations à utiliser les territoires de chasse " (Feit 1991a : 246). Plus encore, les chercheurs montrent dans cette veine, comment le maintien des valeurs de respect et de partage dans la chasse est une question d'affirmation politique, les Cris soulignant qu'ils ne chassent ni ne trappent comme le font les Blancs. Simultanément, cette analyse anthropologique du contexte politique et économique a été influencée en montrant non seulement ce qu'étaient le système des droits de chasse cris et les responsabilités, en résistant à l'imposition d'un cadre néolibéral, mais elle a également ancré leurs revendications politiques et leurs actions. En effet, selon Scott, " alors que les Cris participent davantage au reste du monde, ils font ainsi en utilisant quelques relations clés autochtones » (1984: 77). Feit a poursuivi pour démontrer que " chasser n'est pas seulement une activité centrale pour les Cris, ni simplement un ensemble de connaissances ou une activité spirituelle. Chasser est une expérience continue de vérité comme le pouvoir au cours des vies humaines et dans le monde social dans lequel ils vivent »(Feit $2004:$ 106). Selon lui, la notion crie de pouvoir relève de la réciprocité et du respect entre les partenaires - les animaux, les chasseurs, les gouvernements et même les promoteurs. Tandis qu'ils craignent les comportements irrespectueux et la capacité de destruction de certains acteurs industriels - une tendance qu'ils associent au personnage d'Atuush qui 
est intéressé, asocial et cannibale (Scott 1989 ; Feit 2000) -, le Cri cherche toujours des partenariats avec la société canadienne dominante. Les deux auteurs questionnent la thématique de réciprocité durable et la nature inclusive des Cris : à partir de l'exemple de la générosité animale, ils voient cela comme une responsabilité de partager leurs terres et leurs ressources - une position qui a créé des enchevêtrements complexes et croissants, qui font l'objet de ce numéro spécial. Avant de considérer les développements les plus récents sur le sujet, nous décrirons la situation des voisins algonquiens des Cris. Durant ces décennies d'exploration ethnographique de la territorialité des Cris, que savions-nous des TCF algonquiens au sud d'Eeyou Istchee ${ }^{18}$ ? Il est vrai que la recherche universitaire menée sur la relation entre ces groupes algonquiens et leurs territoires a été considérablement moins volumineuse pour une période et c'est seulement après 2000 qu'un regain d'intérêt ethnographique a émergé. Cette situation peut être expliquée partiellement par la forme très perturbatrice de violence coloniale et l'empiètement territorial auxquels ces groupes ont dû faire face. Situés au sud des Cris, non seulement leur rencontre avec les Euro-Canadiens a eu lieu plus tôt, mais ils ont également été dépossédés de leurs terres plus violemment, en particulier après les années 1950 à cause d'une industrie forestière effrénée aux côtés des développements conjoints, miniers et hydroélectriques, qui les a forcés à altérer ou abandonner leur mode de vie nomade (Poirier 2010). À l'inverse des Cris dont les revendications ont été négociées et partiellement reconnues, leurs droits ont été systématiquement niés et leurs revendications sont largement restées sans réponse jusqu'à aujourd'hui. Par conséquent, et face aux efforts d'assimilateurs variés, ils ont subi une forte baisse des activités de chasse et de trappe, et leurs pratiques quotidiennes sur le territoire ont indubitablement été menacées. Pour ces raisons, il n'est pas surprenant que la plupart des recherches sur ces groupes fussent menées dans une perspective historique : depuis la période des premiers contacts (Clermont 1977 ; Viau 1995 ; Gélinas 2000 ; Chamberland et al. 2004) jusqu’à la dépossession de leurs terres (Leroux et al. 2004 ; Gélinas 2003), les missions catholiques et les pensionnats autochtones (Bousquet 2002, 2005, 2012). Pourtant, même face à autant de défis, leur relation à la terre perdure et ils font d'importants efforts pour maintenir la transmission des pratiques de chasse, des connaissances et des valeurs. D'autant plus que celles-ci sont devenues des sujets politiques et sociaux importants dans leur lutte pour leurs droits et leur souveraineté.

On peut également noter que l'intérêt pour la territorialité et l'utilisation des terres a partiellement été modifié au cours des trois dernières décennies du $\mathrm{XX}^{\mathrm{e}}$ siècle, passant des débats académiques à de la recherche appliquée. En effet, suivant le lancement de la politique fédérale sur les Revendications Territoriales globales $(1973)^{19}$, les Anishinabeg et les Atikamekw (les Innus également) ont travaillé avec des équipes de chercheurs variées pour partager des données relatives à l'utilisation historique et contemporaine des terres ${ }^{20}$. Il en résulte - en lien également avec les recherches récentes sur la territorialité ${ }^{21}$ - que la plupart de ce que nous avons décrit sur les TCF des Cris est partiellement vrai pour les Anishinabeg et les Atimakew, y compris en ce qui concerne les pratiques de subsistances affaiblies. En effet, la gestion collective de la terre, l'éthique de réciprocité, aussi bien que le lien spirituel avec les animaux sont des aspects inhérents de leurs relations territoriales (Poirier 2001). Décrivant ensuite le rôle des chefs de chasse ( $k a$ nikaniwitc), Éthier identifie une série de règles et de pratiques équivalentes à ce que Scott ou Feit ont décrit pour les Cris : gestion des ressources, partage de la nourriture sauvage avec des réseaux étendus, système d'invitation à chasser sur leurs terres, et autorité non coercitive (Éthier $2014: 50-52$ ). Il décrit la façon dont ces pratiques sont intégrées au nehirowisi pimatisiwin, un mode de vie central pour le maintien du bien-être des Atikamekw, de leur langue et de leurs structures sociales. Au-delà de ces similarités, nous devons également souligner une différence significative pour dresser un portrait nuancé des variations dans les régimes fonciers (et une comparaison appropriée). Nous avons déjà mentionné que les TCF des Cris étaient relativement uniques par rapport à leurs relations avec les politiques de conservation mises en place durant la première moitié du XXe siècle. En effet, sur les terres cries, la création des territoires de trappe enregistrés (une évolution des réserves de castors) était basée sur les territoires de chasse des familles qui existaient préalablement (Feit 2005a : 275-276). Par conséquent, même s'ils ont apporté leur juste part de transformation, ils n'ont pas imposé un modèle de régime foncier exogène. Comme l'a décrit Toby Morantz :

\footnotetext{
Une juxtaposition de la carte de John Cooper et des territoires de chasse de Fort George en 1932, conçue dans l'aire de préservation antérieure aux castors, avec une carte des lignes de trappe enregistrées, réalisée par les cris en 1977, montre des limites similaires à ces terres plutôt que le passage de quarante ans et les niveaux d'ingérence extérieure dans leurs stratégies de subsistance.
}

De même, Adrian Tanner qui recherchait des territoires de chasse à Mistassini, dans les années 1960, a vu 
une continuité et un recoupement avec ceux tracés par Speck dans les années 1920. Ce qui a changé était la nature formelle des territoires de chasse des familles. Ce qui avait été auparavant coutumier et flexible, en fonction des circonstances familiales, était maintenant rigide et soumis à la disposition du commerçant ou du gouvernement officiel (Morantz 2002 : 172-173). Du fait de ces modifications, ce que Morantz souligne ici est au cœur de la singularité des territoires de chasse des familles cries. Ce chevauchement est également démontré par l'interchangeabilité des termes utilisés par les Cris pour décrire leurs régimes fonciers : nituuhuи aschii, « territoire de chasse » ou « ligne de trappe »; nituuhuu uuchimaau, " chef de chasse » ou tallyman. Cependant, parmi les Anishinabeg et les Atikamekw, l'histoire ne s'est pas déroulée de la même manière. En effet, pour des raisons particulières et selon la bienveillance de l'agent de conservation, les lignes de trappe enregistrées ne concordaient pas souvent avec les TCF préexistants. Dans les territoires cris y compris, Scott et Morrison (2004, 2005) montrent que des impératifs tels que la frontière administrative entre le Québec et l'Ontario pourraient outrepasser le principe de reconnaissance des frontières autochtones. Mais les Anishinabeg, les Atikamekw et les Innus, alors soumis au "même " système administratif de réserves de castors et de lignes de trappe enregistrées, subissaient davantage de grands bouleversements dus à la colonisation agricole dans les régions de l'Abitibi et du Lac-Saint-Jean, dus également aux plus vastes projets hydro-électriques, aux projets forestiers industriels et miniers, et à l'irruption de la chasse sportive et d'un intérêt pour la pêche. Il en résulte aujourd'hui, pour les peuples algonquiens les plus au sud, que le système de ligne de trappe et que le tallyman ne correspondent pas aux TCF traditionnels ni au modèle de chef de chasse. D'une part, les limites des lignes de trappe rejoignent rarement les territoires de chasse initiaux et, d'autre part, une " appropriation » individuelle de territoires par des hommes adultes s'est imposée au détriment des droits collectifs par l'intermédiaire des chefs de famille. Dans certains cas, il en résulte la disparition des TCF au profit des lignes de trappe administrées à distance, avec des impressions variables de dissonance culturelle. Quand les Anishinabeg de Barrière Lake se sentent étrangers à leur système de territorialité (Pasternak 2013 : 132-139), les Atibitibiwinik ont accepté les points positifs et négatifs de ce droit de propriété établi (Bousquet 2005 : 68). Dans le même ordre d'idées, Leroux note que pour les gens de Kitcisakik, les « lots de piégeage » contemporains qu'il a cartographiés en 1999, même s'ils sont différents des TCF initialement cartographiés par Davidson, sont vus comme un compromis face à la perte territoriale et à l'expansion démographique (Leroux et al. 2004 : 120-130). Dans le cas des Atikamekw, ce processus historique a déjà donné naissance à d'autres résultats : il a mené, jusqu'à présent, à la cohabitation des deux systèmes (Wyatt 2004). En effet, le système Atikamekw d'organisation territoriale ne doit pas être confondu avec les lots de trappe tels que définis dans la protection du castor qui a été établie par le gouvernement provincial en 1951. Bien que les lots soient généralement basés sur les territoires familiaux (tels qu'ils ont été interprétés par les officiers du gouvernement à l'époque), ils ne sont pas équivalents. Les participants de cette étude se sont fréquemment référés à natoho aski et natoho meskano, plutôt qu'à des lots de trappe numérotés. Cette information montre que les Atikamekw continuent d'utiliser leur propre système d'organisation territoriale et que ce système coexiste avec les permis forestiers et les régimes fonciers établis par le gouvernement (Wyatt 2004 : 186).

Ces descriptions et ces nuances dans la cohabitation contemporaine des systèmes fonciers démontrent que les TCF des Algonquiens sont enchevêtrés dans des formes variées de territorialité qui doivent être comprises comme telles. La littérature contemporaine sur le vieux débat nous invite à examiner ces enchevêtrements et c'est ce qui a motivé ce numéro spécial.

\section{Les enchevêtrements complexes des territoires de chasse familiaux au $\mathrm{XXI}^{\mathrm{e}}$ siècle}

Nous n'avons délibérément pas inclus dans l'analyse précédente les contributions les plus récentes sur la territorialité des Algonquiens. Depuis le changement de siècle et particulièrement au cours des dix dernières années, l'étude des TCF est déjà entrée dans une nouvelle phase, selon nous. Cette phase n'est certainement pas en rupture avec le passé récent ni ne s'oppose aux descriptions susmentionnées des TCF. Ce nouveau corpus en tire plutôt partie pour décrire et débattre de plus récents changements qui opèrent sur le terrain. Par exemple, les récentes améliorations des « territorialités entremêlées » concernent davantage les changements en jeu dans les mondes des Algonquiens que de nouvelles articulations théoriques. Ces deux développements, puisqu'ils ont donné l'impulsion à ce numéro spécial, feront l'objet du chapitre final.

Pour les Cris d'Eeyou Istchee, le changement de siècle a été la scène d'un important - et inattendu changement d'évènements. En effet, après des années d'opposition des firmes et de refus de tout développement hydro-électrique sur leurs terres - une saga dont l'évènement marquant a été la décision de 1994 par le 
gouvernement du Québec de reporter sans fin le projet hydro-électrique de Grande Baleine -, le Grand Conseil des Cris a annoncé en 2001 la signature d'une entente de principe avec le gouvernement provincial et HydroQuébec. Cette entente sera connue, après sa signature finale en février 2002, comme " la Paix des Braves " (PDB). Estampillée comme un partenariat « de nation à nation ", elle assure aux Cris un revenu garanti de 3,5 billions de dollars canadiens pour les cinquante prochaines années. Cette entente redéfinit également leur rôle dans l'exploitation industrielle de leurs terres, tout en consentant à la construction d'un nouveau complexe hydro-électrique sur les rivières Eastmain et Rupert (voir la carte dans ce numéro). De plus, la PDB avait pour but de remédier à des déficiences de la Convention de la Baie-James et du Nord québécois en ce qui concerne la gestion forestière - un secteur perçu comme hors de contrôle et comme une source majeure de tensions à travers les communautés cries au sud. Pour résumer l'esprit de cet accord, soulignons, d'après Feit, que « l'augmentation de la population crie a rendu les Cris profondément inquiets au sujet de la création d'emplois pour le nombre croissant de jeunes qui ne font pas de la chasse leur principale activité productrice, bien que la pratique de la chasse maintient une part importante de leurs vies collectives » $(2010: 74)$. Ce n'est pas surprenant que la PDB ait eu d'importantes conséquences sur les territoires de chasse familiaux et qu'elle ait contribué à redéfinir le rôle du tallyman cri, particulièrement dans les communautés touchées par le nouveau projet hydro-électrique - principalement Mistissini, Nemaska et Waskaganish -, ainsi que celles affectées par le nouveau régime forestier - surtout Waswanipi, Ouje-Bougoumou et Mistissini. Les TCF étant maintenant utilisés comme structure pour organiser la gestion du territoire, l'indemnisation, l'emploi et les opportunités entrepreneuriales, tout en respectant les activités économiques variées issues de l'industrie, de nombreux tallymen ont vu leur rôle de chasseur et de porte-parole familial / communautaire redéfini au profit de ceux de gestionnaires de petits-business et de responsables de construction. Bien que cette diversification ne soit pas complètement nouvelle pour eux, elle prend néanmoins de nouvelles proportions. Au même moment, les maîtrestrappeurs et autres chasseurs séniors sont devenus consultants pour des études d'impacts sociaux et environnementaux (voir Nasr et Scott 2010), ainsi que les récipiendaires de diverses mesures de mitigation. Quinze ans après la signature de la Paix des Braves, ce numéro spécial apparait comme l'occasion parfaite de renouveler la description et l'analyse des TCF dans ce contexte difficile. Un changement partiel de perspective semble émerger. En effet, analyser le changement de rôle des tallymen cris et les TCF comme des lieux de résistance culturelle, comme décrits ci-dessus, ne permet plus de saisir pleinement la complexité des enjeux. Harvey Feit lui-même nous invite à considérer un tel changement dans un article de 2010. Il observe :

\begin{abstract}
Il y a des projets autochtones qui ne sont pas centrés sur une opposition au néolibéralisme en général et qui ne cherchent pas à le remplacer à une échelle transnationale. Les Peuples autochtones n'empêchent pas non plus de développer des engagements avec des États-nations néolibéraux ou les marchés n'acceptent pas forcément le néolibéralisme comme une vision ou n'échouent pas à voir les impacts les moins évidents qu'ils peuvent avoir. Ces relations représentent un défi à analyser (Feit $2010: 52$ ).
\end{abstract}

Avant d'analyser les réponses anthropologiques à ces défis, décrivons les enjeux - légèrement différents qui interviennent pour les Atikamekw et les Anishinabeg à cette même époque, tels qu'ils sont décrits dans une série de contributions récentes (pour la liste complète, voir la note de bas de page numéro 19). Dans ces cas-là, plutôt que de mettre en œuvre une entente signée, le centre d'attention est porté sur l'obtention d'un accord, en premier lieu. En effet, comme nous l'avons mentionné plus haut, ces deux nations négocient avec les gouvernements depuis plusieurs décennies maintenant ${ }^{22}$ et la vie de leurs membres a été brusquement affectée par le processus de longue haleine de préparation et de négociation des revendications territoriales. Toutefois, ces négociations exercent une pression sur les TCF puisqu'ils sont l'un des fondements du processus de négociation. Ils sont non seulement au centre des preuves requises de l'utilisation historique et continue de leurs terres (ce qui peut générer des tensions ; voir Bousquet 2005), mais ils servent également de base pour leur modèle de gouvernance et de gestion, faisant ainsi l'objet de débats et de redéfinitions. Ces dernières années, les Atikamekw ont entrepris de renforcer le rôle et l'autorité de leurs chefs de chasse ( $k$ a nikaniwitcik) pour développer leurs propres structures de prise de décision, au regard de la gestion des ressources (Éthier 2014). L'enjeu est de " décrire la façon dont les Nehirowisiwok voient le rôle contemporain du ka nikaniwitc (chef de chasse) tout en comprenant comment des accords de cogestion entre l'État et les Premières Nations pourraient permettre une intégration des institutions traditionnelles comme le $k a$ nikaniwitc dans le processus de décision en ce qui concerne leurs terres » (Houde $2014: 23$ ). Quand, dans ce cas, le stress porte davantage sur les différences de politiques, Poirier nous rappelle que ce processus n'est pas exempt d'enchevêtrements complexes depuis que 
les territoires familiaux des Nehirowisiw, en tant qu'espaces post- et néocoloniaux, sont ainsi devenus les lieux de coexistences, de négociations et d'enchevêtrements complexes entre des régimes de valeurs, des systèmes de régimes fonciers, des formes de gouvernances, des conceptions des territoires forestiers et ses habitants non humains autochtones et non autochtones (Poirier 2017 : 214).

Loin de la perception des observateurs externes selon laquelle les TCF sont obsolètes et sont une mode anthropologique inutile, les régimes fonciers anishinabeg sont au cœur de nombreux défis. Il est vrai toutefois que cette suite d'évènements appelle de nouveaux raffinements dans l'étude anthropologique des TCF des Algonquiens, comme cela a été vu avec l'émergence récente du concept de "territorialité enchevêtrée ", un projet défini dans la publication d'un nouvel ouvrage :

Comprendre comment les enchevêtrements sont vécus dans les différentes parties du monde peut éclairer la façon dont les savoirs et les pratiques de gestion des Autochtones sont remodelés par les rencontres avec la modernité, le néolibéralisme, les oppositions réifiées entre Autochtones et non-Autochtones, et par la proximité des autres pratiques et les obligations envers les terres coutumières (Poirier et Dussart 2017 : 4).

Notre numéro spécial s'aligne sur ce projet, puisque nous analysons les TCF comme l'un des éléments en jeu sur les terres algonquiennes, incluant la résistance des pratiques de chasse, l'économie globalisée de l'extraction des ressources, les négociations et les règlements des revendications territoriales, la coexistence difficile des ontologies relationnelles et naturalistes, les dynamiques culturelles et l'affirmation politique. Par conséquent, ce numéro reprend la description des dynamiques profondément troublantes des pratiques néocoloniales canadiennes. Comme Nadasdy l'a décrit :

La négociation et la mise en œuvre de l'entente des revendications territoriales représentent une tentative d'intégrer la relation unique à la terre des Peuples autochtones dans les institutions existantes, légales et politiques, de l'État canadien. [...] De plus, le simple fait de négocier ces ententes, apparemment sans faire mention de leur mise en œuvre - requiert la création de structures gouvernementales et agit au sein des communautés des Premières Nations elles-mêmes, qui sont de loin les plus compatibles avec les styles de vie des bureaucrates euro-canadiens qu'avec ceux des chasseurs et des trappeurs des Premières Nations. (Nadasdy 2003 : 223-224).

C'est d'autant plus vrai dans les cas décrits par les auteurs de ce numéro. Cependant, d'autres dynamiques sont également en jeu et selon nous, une notion comme l'enchevêtrement territorial permet d'embrasser non seulement les obstacles ontologiques, mais aussi les tentatives des acteurs autochtones pour les dépasser et trouver, pour leurs terres, une façon éthique d'inclure le sens des responsabilités à d'autres êtres pour exister dans ce contexte complexe ${ }^{23}$. D'ailleurs, comme l'a relevé plus haut Harvey Feit, cela nous permet également de comprendre les multiples projets des Peuples autochtones qui incluent ceux qui cherchent à intégrer les volets de l'exploitation globale de ressources. Selon notre opinion dans ce numéro, les TCF algonquiens peuvent être décrits à la fois en tant qu'outils de résistance et d'espace de changement et d'adaptation (Papillon 2012 ; Salée et Lévesque 2010). L'enjeu est la capacité, tant pour les individus que pour les communautés, de maintenir et de créer des institutions de régimes fonciers appropriés pour assurer leur santé sociale et culturelle, ainsi que leur développement économique.

Ainsi, les contributions de ce numéro visent à délimiter plus précisément les dimensions, complexes et dynamiques, de la rencontre permanente entre les territoires de chasse familiaux des Algonquiens et la société colonisatrice ; un projet qui élude précisément le débat classique. Ce faisant et dans l'esprit du numéro spécial publié il y a trente ans, nous aspirons à créer un espace de dialogue entre les différentes générations de chercheurs qui travaillent sur ce sujet. Nous commençons avec une Introduction de Philip Awashish, un négociateur clé de la CBJNQ et un expert des structures sociales et culturelles de sa nation, et terminons le numéro avec le frontispice de Jasmin Habib et les documents qu'elle a assemblés, lesquels nous rappellent les principes fondamentaux de la gouvernance et des régimes fonciers cris. Dan l'avant-propos, Morantz présente une rétrospective sur la manière dont le débat original s'est déroulé parmi les anthropologues et les ethnohistoriens, Awashish - un négociateur clé de la CBJNQ et un expert des structures sociale et culturelle de sa nation - nous rappelle les principes fondamentaux des régimes fonciers cris et leur importance pour les Cris. Ensuite, plusieurs articles touchent à l'essence même des divers enjeux et contraintes contemporains dans lesquels les territoires algonquiens sont enchevêtrés. Éthier et Poirier démontrent comment, dans le contexte des revendications territoriales, le paysage forestier (notcimik) est un lieu d'intrication complexe entre les pratiques de chasse, les systèmes de valeurs, les régimes fonciers, les formes de gouvernance et les conceptions des habitants non humains autochtones et non-autochtones. Puis, Inksetter explique que les sources archéologiques peuvent être utilisées pour (re)discuter des origines des TCF. En exploitant ces données dans 
un contexte contemporain, elle montre comment les enjeux politiques, accrus par les négociations des revendications territoriales, influent la perspective des Anishinabeg en ce qui a trait à cette question des origines. Les trois articles suivants traitent $d u$ changement de contexte des Cris. Chaplier analyse comment, à Nemaska, la notion de "propriété comme partage " a émergé à partir du changement de rôle du maître-trappeur et des TCF dans le contexte de la Paix des Braves. En évoquant les changements en cours dans le cadre du projet hydro-électrique d'Eastmain-Rupert, elle décrit la manière dont les familles et les utilisateurs des terres ont influencé et recadré les pressions exercées à la privatisation pour répondre à l'éthique de partage si chère aux Cris. Scott examine les relations dynamiques de l'organisation territoriale au niveau de la bande et de la famille, un aspect relativement négligé dans la littérature. Son article montre comment les dimensions du droit de propriété coutumier, au niveau familial et au niveau de la bande, ont été complémentaires et intégrées l'un à l'autre au cours de l'histoire. La contribution de Lessard ouvre de nouvelles perspectives théoriques, en analysant une forme d'enchevêtrement largement occultée jusqu'à présent dans la région : l'imbroglio du chevauchement des revendications territoriales. En effet, à partir de la création de la communauté de Washaw Sibi Eeyou, il analyse le rôle de la cartographie des TCF historiques dans les dynamiques complexes des politiques ethniques de la communauté. Il démontre également l'intrication des identités et des vies des Cris et des Anishinabeg dans la région. Comme Sylvie Poirier avance dans un article récent (2017), ce type d'enchevêtrement qui est répandu dans les terres des Algonquiens a été rarement analysé et mérite notre attention. Puis, ayant soigneusement assemblé diverses lettres écrites par des chefs et des chasseurs cris, Habib clôt le numéro avec les voix de la résistance et de la détermination autochtone qui résonnent dans le présent. Enfin, nous le plaisir de lire les commentaires de Feit et Tanner qui offrent leur réflexion sur ces transformations récentes, au regard de leur longue familiarité avec le débat sur les TCF, tout en faisant le lien entre le passé et le présent.

Mélanie Chaplier, département d'anthropologie, McGill University, Montréal, QC.Courriel:melaniechaplier@ gmail.com.

Colin Scott, département d'anthropologie, McGill University, Montréal, QC. Courriel : colin.scott@mcgill.ca.

\section{Notes}

1 Le mot "Algonquien " se réfère à un large groupe sociolinguistique historique, étendu depuis la majeure partie de l'est du Canada et de la Nouvelle-Angleterre jusqu'aux
Rocheuses canadiennes et au Midwest américain. Dans son utilisation contemporaine, le terme inclut habituellement plusieurs Premières Nations du Québec et de l'Ontario (Atikamekw, Innu, Algonquin, Cri, Micmac, Malécite, Ojibway). Dans ce numéro, nous avons partagé les contributions des groupes occupant la partie nord-ouest du Québec (avec quelques chevauchements en Ontario) : les Atikamekw Nehirowisiwok, les Cris d'Eeyou Istschee et les Algonquins/Anicinabe de l'Abitibi et de l'Ontario). Veuillez noter la différence, qui prête parfois à confusion, entre les termes "Algonquien » (groupe sociolinguistique élargi) et « Algonquin » (désignant les dix communautés anicinabe).

2 À l'exception de l'article de Leila Inksetter dans lequel elle se réfère aux données archéologiques pour invalider l'hypothèse d'un changement de régime alimentaire des Algonquins (des grands animaux aux castors à fourrure) un argument souvent utilisé pour appuyer la nature postcontact des TCF.

3 Selon Siomonn Pulla (2003, 2006, 2008), de même qu'Harvey Feit (1991a, 2005) et Alfred I. Hallowell (1951), il y a eu une déformation quant aux motivations de Speck à décrire les TCF comme autochtones en premier lieu. Alors qu'un intérêt théorique était en jeu, sa véritable raison était son plaidoyer pour les droits des Autochtones. Sa relation avec les Autochtones relevait de l'amitié, et il était convaincu que son travail pourrait les aider. Il pensait que la société colonisatrice serait enthousiaste (ou simplement obligée) à reconnaître les revendications territoriales autochtones s'il pouvait démontrer que les colons avaient effectivement développé une forme de relation de propriété avec ces territoires. Néanmoins, le positionnement théorique de Speck est ce qui a rendu son travail remarquable pour l'anthropologie canadienne (et américaine) naissante, une réalité qui nous informe au sujet de notre discipline au début du $\mathrm{XX}^{\mathrm{e}}$ siècle.

4 Dans une tentative pour mieux saisir l'« altérité » des TCF et des relations des Autochtones à la terre, Tanner formulait dans un premier article (1971 : 81-82) une hypothèse intéressante. Notant que l'un des termes utilisés par les Cris pour désigner leur TCF était nimeskanu (« mon chemin, ma voie ") et que leur description était plutôt liée aux animaux eux-mêmes qu'aux points géographiques, il affirma que les TCF seraient mieux compris en termes de mouvements (des animaux et des chasseurs sur le territoire) ». Il n'a pourtant pas poursuivi cette nouvelle perspective dans ses écrits ultérieurs.

5 Une exception vient à l'esprit avec le travail d'Alfred I. Hallowell (1949, 1951, 1975) qui était en avance sur son temps avec son étude de l'ontologie ojibway (formulée en tant que « vision du monde »). Un pionnier de la première heure de ce qui deviendra, des années plus tard, le «tournant ontologique », il montra la nature relationnelle de la vision du monde des Ojibway et souligna l'existence d'interactions sociales entre les Ojibway et les personnes autres-qu'humaines (1975).

6 Une généralisation qui s'est avérée particulièrement problématique dans le cas des Innu-Naskapi du Nord-duQuébec et du Labrador. Comme d'autres auteurs l'ont confirmé (Henriksen 1973 ; Tanner 1983 ; Mailhot 1986), les schémas de leur chasse aux caribous extrêmement mobiles ne correspondent pas à la théorie des TCF. Pour 
être juste, une telle observation avait été formulée au début du débat original. Selon Davidson (1928 : 50), au nord de la bande de Mitsissini, « en conséquence de la progressivité dans la pauvreté naturelle comme les contrées de terres arides de la région Ungava sont approchées, il est attendu que les familles propriétaires des localités en assumeront de moins en moins l'importance dans le système économique de ces gens. ". De même, Lips (1947 : 398-399) mentionne que « les tribus nordiques qui, avec des terres non divisées entre des zones de chasse familiale, chassent également le caribou »; et ailleurs (1947:428), il avance que "dans les régions des bandes nordiques, au sud de la circonscription d'Ungava où les troupeaux de caribous abondent, l'institution de séparer les terres de chasse familiale serait inappropriée et le groupe entier, par conséquent, se rassemble pour une chasse au caribou collective. Mais les deux pratiques peuvent exister côte à côte ou même alternativement à certains moments ». Malheureusement, ces nuances ont été partiellement éclipsées par l'évolution dichotomique du débat.

7 Le travail d'Eleanor Leacock (1954) et celui des auteurs qui ont suivi sa perspective (tels que Stewart et Murphy) ont définitivement offert davantage d'analyses nuancées de la colonisation et du changement social. Ils ont montré que les changements rencontraient de la résistance et des réticences à abandonner le mode de vie cynégétique. Néanmoins, le cadre de ces changements comme " acculturation » a eu l'avantage. L'autre question, alors, si nous comparons leur travail à l'anthropologie contemporaine ou aux perspectives qui ont été développées dans d'autres contextes au même moment (voir, par exemple, le travail ethnologique de l'école de Manchester en Afrique), est leur lecture unilinéaire du changement social. Comme Feit a souligné : « ni Stewart ni Leacock n'a considéré la possibilité de la continuité d'une morale distincte, productive et sociale des économies chez les Autochtones contemporains ou dans les sociétés de chasse » (2005b : 62).

8 Il a dirigé le projet de 1964 à 1971. Ensuite, de 1971 à 1976, le projet est devenu, sous le leadership de Richard F. Salisbury, un laboratoire pour l'anthropologie appliquée dans le contexte du développement hydroélectrique de la baie James. Pour plus de détails sur le projet, voir l'entrevue d'Adrian Tanner de la série "Les possédés et leurs modes »: https://www.anthropologie-societes.ant. ulaval.ca/adrian-tanner-film-2-life-trapline-yukon-andmegill-cree-project.

9 Ignatius La Rusic, Adrian Tanner et Harvey Feit, mais aussi Bernard Bernier, Roger Pothier et Marcel Samson.

10 De manière fortuite, le projet McGill-Cree a commencé quelques années avant l'un des plus gros projets de développement de ressources dans l'histoire canadienne, le projet hydroélectrique de la baie James, empiété sur les territoires cris. À l'époque, le choix de se focaliser sur les Cris se fondait sur divers développements en cours dans la région (de nouvelles mines et le boom forestier). Avec la nouvelle voie de chemin de fer et les routes construites dans les années 1960, le sud de la baie James était vu comme une bonne région pour étudier les impacts des nouveaux développements sur les peuples autochtones (Harvey Feit, communication personnelle).
11 Ce « projet du siècle », à l'époque de son annonce, incluait trois complexes régionaux hydroélectriques, alors que ses infrastructures routières devaient stimuler l'expansion de l'industrie minière et forestière à travers les territoires cris.

12 Voir l'édition spéciale publiée dans la revue nouvellement créée Recherches amérindiennes au Québec (1971), dans laquelle le comité éditorial admet que «faisant face aux conséquences potentielles de ce projet, nous devons "dire quelque chose" ... ou, au moins, autoriser ces travaux avec les Cris pour qu'ils s'expriment eux-mêmes » (1971:1).

13 Cela fait en sorte que les TCF des Cris sont relativement uniques parmi les Algonquiens, au sein desquels il existe d'importes disparités selon l'intégration (ou le manque d'intégration) des TCF dans les politiques de conservation.

14 Leurs intérêts pour les pratiques de chasse et la gestion $\mathrm{du}$ territoire variaient. Alors qu'il travaillait sur les manifestations spirituelles et religieuses (2002 [1975]), l'intérêt de Preston pour les activités de chasse était moins direct (la majorité de son travail sur le sujet était effectivement effectué avec les Cris vivant du côté ouest de la baie James ; voir George, Berkes et Preston, 1995). Quant à Tanner, il est le seul à avoir publié une monographie complète sur les pratiques cynégétiques des Cris (1979), tandis que Feit et Scott sont les plus prolifiques en ce qui concerne les évolutions récentes de la société crie, maintenant leur intérêt et leur effort de publication sur le temps présent (Feit 1971, 1989, 1991a, 1991b, 2000, 2004a, 2004b, 2005, 2009, 2017; Scott 1982, 1984, 1997, 2001, 2004, 2013, 2017).

15 Ils n'étaient évidemment pas les seuls à le faire, mais ils sont définitivement les chercheurs les plus prolifiques et les mieux reconnus. À leurs côtés, d'autres ont développé un intérêt temporaire ou ont travaillé sur divers sujets, mentionnons Berkes (1986, 1988), Brelsford (1983), Salisbury (1986), La Rusic (1968), Désy (1968), Craik et Casgrain (1986), Knight (1968), et plus récemment Carlson (2004, 2008), Niezen (1993, 1998), Mulrennan (2015), Atkinson et Mulrennan (2009), Desbiens (2004, 2008, 2013a, 2013b), Adelson (2000), Gagné (1994), Craik (2004), Whiteman (1998, 2004) et Susan Preston (2011).

16 Si nous définissons la propriété dans un sens strict. Pour une perspective plus détaillée sur le débat au sujet de la propriété, voir Chaplier (2014 et ce numéro).

17 À partir d'une perspective marxiste, Tanner voulait « montrer que la complexité fondamentale des relations sociales, ce que j'ai appelé le mode de production, n'est pas simplement une fonction directe des conditions matérielles, mais est une construction à travers laquelle les idées et les symboles de l'environnement matériel sont mis en action » $(1979: 43)$.

18 Pour diverses raisons, nous avons malheureusement été incapables d'inclure le cas Innu (Montagnais) dans ce numéro. Cependant, pour celles et ceux intéressés à leur relation contemporaine à leurs terres, voir par exemple, Armitage (1990), Charest (1995, 1996), Lacasse (1996, 2004), Mailhot (1993), Mailhot and Vincent (1980), Tanner (1983) et Samson (2003).

19 Une politique qui n'a pas vraiment été fructueuse pour les groupes algonquiens au Québec. En effet, à l'exception de la CBJNQ, aucun accord n'a abouti. Après des décennies d'études, de rapports et négociations, les Algonquins, les 
Innu et les Atikamekw attendent encore que leurs droits soient reconnus.

20 Parfois inaccessible au public, une description complète de cette littérature est difficile. Certains des rapports les plus compréhensifs incluent : Conseil Atikamekw-Montagnais (1982), Conseil de la Nation Attikamekw Nehirowisiw (1997, 1998), Association Mamo Atoskewin Atikamekw (1994), Dandenault (1983), Poirier et Niquay (1999), Frénette (1988, 1993), Henriksen (1977), Roark-Calnek (1995, 1996) (liste non exhaustive).

21 Pour les Atikamekw, voir Poirier (2000, 2001, 2004, 2008, 2010, 2013), Wyatt (2004), Wyatt et Chilton (2014), Morissette (2007), Houde (2011, 2014), Éthier (2014) et Laurent et Veilleux (2014). Pour les Algonquins, voir Bousquet (2002, 2005), Leroux et al. (2004, 2009) et Pasternak (2013).

22 Ce processus de négociation a débuté peu après pour les Algonquins, pour lesquels la situation est rendue plus complexe à cause des chevauchements avec des accords antérieurs (Traité 9 en Ontario, CBJNQ au Québec ; Bousquet 2005).

23 Un processus complexe que l'idée des enchevêtrements décrit bien. Un autre concept anthropologique adéquat, pas si loin de l'idée de chevauchements, est celui de " friction " décrit par Anna Tsing : "Les routes sont une bonne image pour conceptualiser le fonctionnement d'une friction : les routes créent des passages qui facilitent le mouvement et le rend plus efficace, mais ce faisant, ils limitent là où nous allons. La facilité qu'ils offrent est aussi une structure de confinement. La friction module des trajectoires historiques possibles, exclusives et spécifiques. Les répercussions des rencontres face à la différence peuvent être compromettantes ou encourageantes. La friction n'est pas synonyme de résistance. L'hégémonie est faite et défaite avec la friction. Ce sont ces vicissitudes que j'appelle friction. La friction établit une connexion globale puissante et effective. Entre temps, sans même s'en rendre compte, la friction entre dans la douce opération du pouvoir global » (Tsing 2005 : 6). C'est également une puissante métaphore pour décrire la contemporanéité algonquienne.

\section{Références}

AMAA (Association Mamo Atoskewin Atikamekw), 1994. Rapport Final, Identification et protection de l'usage Atikamekw de la forêt, Phase II. La Tuque.

Adelson, Naomi, 2000. 'Being Alive Well' : Health and the Politics of Cree Well-being. Toronto, University of Toronto Press.

Armitage, Peter, 1990. Land Use and Occupancy among the Innu of Utshimassit and Sheshatshit. Rapport préparé pour la Nation Innu, Sheshatshit, Nitassinan.

Atkinson, Myriam, and Monica Mulrennan, 2009. « Local Protest and Resistance to the Rupert Diversion Project, Northern Quebec », Arctic, 62 (4) : 468-480.

Berkes, Fikret, 1986. "Common Property Resources and Hunting Territories ", Anthropologica, 28 (1-2) : 145-162. . 1988. "Environmental Philosophy of the Chisasibi People of James Bay ». In Freeman et Carbyn (dir.), Traditional Knowledge and Renewable Resource Management in Northern Regions, p. 7-21. Edmonton, Boreal Institute for Northern Studies.
Bishop, Charles, 1986. « Territoriality along Northeastern Algonquians ", Anthropologica, 28 (1-2) : 37-63.

Bousquet, Marie-Pierre, 1999. "Sites ancestraux et territoire chez les Algonquins du Québec ", Canadian Studies, 47 : 29-40.

—. 2002. "Quand nous vivions dans le bois ". Le changement spatial et sa dimension générationnelle: L'exemple des Algonquins du Canada. Thèse de doctorat, département d'anthropologie, Université Laval.

—. 2005. «Des lois, des cartes et de valeurs sociales : Les débats générationnels dans une communauté Algonquine du Québec ». In Wolfart (dir.), Papers of the 36th Algonquian Conference, p. 53-74. Winnipeg, University of Manitoba.

—. 2012. « Êtres libres ou sauvages à civiliser ? L'éducation des jeunes Amérindiens dans les pensionnats indiens au Québec, des années 1950 à 1970 ». In Mathias Gardet et David Niquet (dir.), Enfances déplacées en situation coloniale, p. 163-192. Rennes, Presses universitaires de Rennes.

Brelsford, Taylor, 1983. Hunters and Workers among the Nemaska Cree. The Role of Ideology in a Dependant Mode of Production. " Mémoire de maîtrise, Departement of Anthropology, McGill University.

Carlson, Hans. 2004. "A Watershed of Words : Litigating and Negotiating Nature in Eastern James Bay, 1971-75 ", Canadian Historical Review, 85 (1) : 63-84.

- 2008. Home is the Hunter. Vancouver : UBC Press.

Chance, Norman A. (dir.), 1968. Conflict in Culture Problems of Developmental Change Among the Cree. Ottawa, Canadian Research Centre for Anthropology.

Clermont, Norman, 1977. Ma femme, ma hache et mon couteau croche : Deux siècles d'histoire à Weymontachie. Québec, Ministère des Affaires culturelles.

Cooper, John, 1939. « Is the Algonkian Hunting Ground System Pre-Columbian? », American Anthropologist, 41 (1) : 66-90.

Chamberland, Roland et al., 2004. Terra Incognita des Kotakoutouemis. L'Alonquinie orientale au XVIIe siècle. Québec, Presses de l'Université Laval.

Chaplier, Mélanie, 2014. « La "Paix des Braves" et la gestion des ressources naturelles chez les Cris de la baie James (Québec). Transformations du territoire et conséquences sur le rôle du tallyman ». In Charlotte Bréda, Mélanie Chaplier, Julie Hermesse et Emmanuelle Piccoli (dir.), Terres (dés)humanisées : Ressources et climat, p. 127-153. Louvain-la- Neuve, Academia Bruylandt.

—. 2018. « Property as Sharing: A Reflection on the Nature of Land Ownership among the Cree of Eeyou Istchee after the 'Paix des Braves ", Anthropologica 60 (1) : 61-75.

Charest, Paul, 1995. « La composition des groupes de chasse chez les Mamit Innuat ». In François Trudel, Paul Charest et Yvan Breton, La construction de l'anthropologie québécoise. Mélanges offerts à Marc-Adélard Tremblay, p. 367-396. Québec, Les Presses de l'Université Laval.

- 1996. "La supposée disparition des Atikamekw et des Montagnais ", Recherches amérindiennes au Québec, 26 (2) : 84-85.

Clermont, Norman, 1977. Ma femme, ma hache et mon couteau croche : deux siècles d'histoire à Weymontachie. Québec, Ministère des Affaires Culturelles. 
Conseil Atikamekw-Montagnais, 1982. Nihastanan nitasinan [Notre terre, nous l'aimons et nous y tenons] : Revendications territoriales des bandes Attikamèques et montagnaises. Conseil Attikamek-Montagnais. Adressées au Ministère des Affaires Indiennes et du Nord, Représentant du gouvernement du Canada, Québec, Conseil de la Nation Attikamekw Nehirowisiw (CNA).

—. 1997. Politique sociale Atikamekw. Québec, Conseil de la Nation Atikamekw.

— 1998. E Nehiromonaniwok ka aicinikateki Atikamekw iriniw kitci Masinahikan otci ka wi orasinahikatek / Version atikamekw des termes utilisés dans la consultation sur la constitution atikamekw. La Tuque, Conseil de la Nation Atikamekw.

Craik, Brian, 2004. " The Importance of Working Together : Exclusions, Conflicts, and Participation in James Bay, Quebec ». In Mario Blaser, Harvey Feit and Glenn McRae (dir.), In The Way of Development. Indigenous Peoples, Life Projects and Globalization, p. 166-186. New York, Zed Books.

Craik, Brian, et Byers Casgrain, 1986. " Making a Living in the Bush : Land Tenure at Waskaganish ", Anthropologica, $28(1-2)$ : 175-186.

Cruikshank, Julie, 1993. " The Politics of Ethnography in the Canadian North ». In James Waldram et Noel Dyck, Anthropology, Public Policy, and Native Peoples in Canada, p.133-145. Montreal, McGill-Queen's University Press.

Dandenault, André, 1983. « Occupation et utilisation du territoire par les Attikameks de Weymontachie ». In D. Brassard et D. Castonguay (dir.), Rapport de recherche dans le cadre du projet sur l'occupation et l'utilisation du territoire. Village des Hurons, Québec.

Davidson, D. Sutherland, 1928. The Family hunting territories of the Grand Lake Victoria Indians, Proceedings of the $22^{\text {nd }}$ International Congress of Americanists, 2. p. 69-95. 1926, Rome.

Deloria, Vine Jr., 2004. « Philosophy and Tribal Peoples ». In Anne WATERS (dir.), American Indian Thought, p. 3-12. Malden, Blackwell Publishing.

Desbiens, Caroline, 2004. « Nation to Nation : Defining New Structures of Development in Northern Quebec ", Economic Geography, 80 (4) : 351-366.

—. 2008. « Le Jardin au Bout du Monde : Terre, texte et production du paysage à la Baie James ", Recherches amérindiennes au Québec, 38 (1) : 7-15.

- 2013a. Power from the North. Territory, Identity, and the Culture of Hydroelectricity in Quebec. Vancouver, UBC Press.

- 2013b. « From Passive to Active Dialogue? Aboriginal Lands, Development and Metissage in Quebec, Canada ", Cultural Geographies, 21 (1) : 99-114.

Descola, Philippe, 2005. Par-delà nature et culture. Paris, Gallimard.

Désy, Pierrette, 1968. Fort-George ou Tsesa-Sippi. Thèse de doctorat, département de géographie, Université de Paris La Sorbonne.

Dussart, Françoise et Sylvie Poirier, 2017. Entangled Territorialities. Negotiating Indigenous Lands in Australia and Canada. Toronto, University of Toronto Press.
Éthier, Benoît, 2014. « Nehirowisiw Kiskeritamowina : Acquisition, utilisation et transmission de savoir-faire et de savoir-être dans un monde de chasseurs ", Recherches amérindiennes au Québec, 44 (1) : 49-59.

Feit, Harvey, 1971. « L'ethno-écologie des Cris Waswanipis, ou comment des chasseurs peuvent aménager leurs ressources ", Recherches amérindiennes au Québec, 1 (4-5) : 84-91.

- 1982. "The Future of Hunter within Nations-States : Anthropology and the James Bay Cree ». In E. Leacock et R. Lee (dir.), Politics and History in Band Societies, p. 373-411. Cambridge, Cambridge University Press. _ 1988. "Waswanipi Cree Management of Land and Wildlife : Cree Ethno-Ecology Revisited ». In B. Cox (dir.), Native Peoples, Native Lands : Canadian Indians, Inuit and Metis, p. 75-91. Ottawa, Carleton University Press.

_ 1989. " James Bay Cree Self-Governance and Land Management ». In E. Wilmsen (dir.), We are Here : Politics of Aboriginal Land Tenure, p. 68-98. Los Angeles, University of California Press.

—. 1991a. " Gifts of the Land : Hunting Territories, Guaranteed Incomes and the Construction of Social Relations in James Bay Cree Society ", Senri Ethnological Studies, 30 : 223-268.

. 1991b. « The Construction of Algonquian Hunting Territories. Private Property as Moral Lesson, Policy Advocacy, and Ethnographic Error ». In George W. Stocking (dir.), Colonial Situations : Essays on the Contextualization of Ethnographic Knowledge, p. 109-134. Madison, University of Wisconsin Press.

- 2000. "Les animaux comme partenaires de chasse : Réciprocité chez les Cris de la Baie James », Terrain, 34 : 123-142.

- 2004a. « James Bay Crees' Life Projects and Politics : Histories of Place, Animal Partners and Enduring Relationships ». In Mario Blaser, Harvey Feit et Glenn McRae (dir.), In the Way of Development : Indigenous Peoples, Life Projects and Globalization, p. 92-110. Londres, Zed Books.

- 2004b. « Les territoires de chasse algonquiens avant leur 'découverte' ? Études et histoires sur la tenure, les incendies de forêt et la sociabilité de la chasse ", Recherches amérindiennes au Québec, 34 (3) : 5-21.

- 2004, [1995]. «Hunting and the Quest for Power : the James Bay Cree and Whiteman Development ». In Bruce Mossiron et Roderick Wilson (dir.). Native Peoples. The Canadian Experience, p. 101-128. Toronto, Oxford University Press.

. 2005. « Re-Cognizing Co-Management as Co-Governance : Histories and Visions of Conservation at James Bay ", Anthropologica, 47 (2) : 267-288.

- 2009a. "Histories of the Past, Histories of the Future :

The Committed Anthropologies of Richard Slobodin,

Franck G. Speck, and Eleanor Leacock ». In Richard J. Preston (dir.), A Kindly Scrutiny of Human Nature : In Honour of Richard Slobodin, p. 45-76. Waterloo, Wilfrid Laurier University Press.

_. 2009. «Governmental Rationalities and Indigenous co-governance : James Bay Cree coexistence, from Mercantilist Partnerships to Neoliberal Mechanisms ». In S. Bernstein et W. Coleman (dir.), Unsettled legitimacy. 
Political community, Power and Authority in a Global Era, p. 97-128. Vancouver, UBC Press.

_ 2010. « Neoliberal Governance and James Bay Cree

Governance : Negotiated Agreements, Oppositional

Struggles, and Co-governance ». In M. Blaser, R. De Costa,

D. McGregor et W. Coleman (dir.), Indigenous People and

Autonomy. Insights for a Global Age, p. 49-79. Vancouver,

UBC Press.

—. 2017. «Dialogues on surviving : Eeyou hunters' ways of

engagement with land, governments and youth ». In

Françoise Dussart et Sylvie Poirier (dir.), Entangled

Territorialities. Negotiating Indigenous lands in

Australia and Canada, p. 25-50. Toronto, University of

Toronto Press.

Feit, Harvey et Robert Beaulieu, 2001. "Voices from a

Disappearing Forest : Government, Corporate and Cree

Participatory Forestry Management Practices ». In Colin

Scott (dir.), Aboriginal Autonomy and Development in

Northern Quebec and Labrador, p. 119-148. Vancouver,

University of British Columbia Press.

Frénette, Jacques, 1988. Le pays des Anicinabe. La revendication territoriale globale de la nation Algonquine. Enoncé de revendication documenté et rédigé par le conseil de Bande, Réserve Algonquine de Maniwaka, Miméo.

_ 1993. «Kitigan Zibi Anishinabeg : le territoire et les activités économiques des Algonquins de la rivière du désert (Maniwaki), 1850-1950 ", Recherches amérindiennes au Québec, 23 (2-3) : 39-51.

Gagné, Marie-Anik, 1994. A Nation within a Nation. Dependency and the Cree. Montreal, Black Rose Books.

George, Peter, Fikret Berkes, et Richard Preston, 1995.

" Aboriginal Harvesting in the Moose River Basin : A Historical and Contemporary Analysis ", Canadian Review of Sociology and Anthropology. La Revue Canadienne de Sociologie et d'Anthropologie, 32 (1) : 69-90.

Gélinas, Claude, 2000. La gestion de l'Étranger. Les Atikamekw et la présence Eurocanadienne en Haute Mauricie, 1\%60-18\%0. Sillery, Éditions du Septentrion.

- 2003. Entre l'assommoir et le godendart. Les Atikamekw et la conquête du Moyen - Nord québécois, 18\%0-1940. Sillery, Editions du Septentrion.

Gouvernement du Québec, 1975. The James Bay and Northern Quebec Agreement. Quebec, Government of Quebec.

Hallowell, A. Irving. 1949. "The Size of Algonkian Hunting

Territories : A Function of Ecological Adjustment », American Anthropologist, 51 : 35-45.

- 1951. «Frank Gouldsmith Speck, 1881-1950», American Anthropologist, 53 (1) : 67-87.

- 1975. "Ojibwa Ontology, Behaviour, and World View ». In Dennis Tedlock and Barbara Tedlock (dir.), Teachings from the American Earth, p. 141-179. New York, Liveright.

Hedican, Edward, 2008. Applied Anthropology in Canada: Understanding Aboriginal Issues. Toronto, University of Toronto Press.

Henriksen, Georg, 1973. Hunters in the Barrens : The Naskapi on the edge of the White man's world. St. John's, ISER.

- 1977. Land Use and Occupancy among the Naskapi of Davis Inlet. Unpublished report for the Naskapi Montagnais Innu Association.
Houde, Nicolas, 2011. "Experimenting with what will become our traditions": Adaptive comanagement as a bridge to an Atikamekw Nehirowisiw post-treaty world in Nitaskinan, Canada. Thèse de doctorat, Department of Geography, Montreal, McGill University.

— . 2014. « La gouvernance territoriale contemporaine du Nitaskinan : Tradition, adaptation et flexibilité ", Recherches amérindiennes au Québec, 44 (1) : 23-33.

Ingold, Tim, 2000. The Perception of the Environment. Essays in Livelihood, Dwelling and Skill. New York, Routledge.

Jenness, Diamond, 1935. The Ojibwa Indians of Parry Island. Their Social and Religious Life. Coll. Anthropological Series $n^{\circ} 17$, bulletin 78, Ottawa, National Museum of Canada.

Knight, Rolf, 1968. Ecological Factors in Changing Economy and Social Organization among the Rupert House Cree. Ottawa, National Museum of Canada, Anthropology Papers, 15.

Lacasse, Jean-Paul, 1996. " Le territoire dans l'univers innu d'aujourd'hui », Cahiers de Géographie de Québec, 40 (110) : 185-204.

- 2004. Les Innus et le territoire. Innu Tipenitamun. Sillery, Éditions du Septentrion.

La Rusic, Ignatius, 1968. The new Auchimau. Mémoire de maîtrise, Departement of Sociology and Anthropology, Montreal, McGill University.

Leacock, Eleanor, 1954. The Montagnais "Hunting Territory" and the Fur Trade. New York, American Anthropological Association, Memoir $\mathrm{n}^{\circ} 78$.

Leroux, Jacques, 2009. «Éthique et symbolique de la responsabilité territoriale chez les peuples algonquiens du Québec ", Recherches amérindiennes au Québec, 39 (1-2) : 85-97.

Leroux, Jacques et al. 2004. Au pays des peaux de chagrin. Occupation et exploitation territoriales à Kitchisakik (Grand-Lac-Victoria) au XXe siècle. Québec, Presses de l'Université Laval.

Lips, Julius, 1947. "Naskapi Law », Transactions of the American Philosophical Society, 37 (4) : 379-492.

Mailhot, José, 1986. "Territorial Mobility among the Montagnais-Naskapi of Labrador.” Anthropologica, 28 (1-2) : 92-107.

—. 1993. Au Pays des Innus. Les gens de Sheshatshit. Montréal, Presses de Recherches amérindiennes au Québec.

Mailhot, José et Sylvie Vincent, 1980. Le discours montagnais sur le territoire. Québec, Conseil des Atikamekw et des Montagnais.

Morantz, Toby, 1978. « The Probability of Family Hunting Territories in 18th Century James Bay : Old Evidence Newly Presented ». In W. Cowan (dir.), Proceedings of the 9th Algonquian Conference, p. 224-236. Ottawa, Carleton University Press.

- 1986. "Historical Perspectives on Family Hunting Territories in Eastern James Bay ", Anthropologica, 28 (1-2) : 64-91.

- 2002. The White Man's Gonna Getcha. Montreal, McGillQueen's University Press.

- 2018. «Foreword: Remembering the Algonquian Family Hunting Territory Debate ", Anthropologica 60 (1) : 10-20. 
Murphy, Robert, and Julian Steward, 1956. « Tappers and Trappers : Parallel Process in Acculturation ", Economic Development and Cultural Change, 4 (4) : 335-355.

Morissette, Anny, 2007. « Composer avec un système imposé : la tradition et le conseil de bande à Manawan ", Recherches amérindiennes au Québec, 37 (2-3) : 127-138.

Mulrennan, Monica, 2015. « Aboriginal Peoples in Relation to Resource and Environmental Management ». In Mitchell, Bruce (dir.), Resource and Environmental Management in Canada : Addressing Conflict and Uncertainty, p. 56-79. Toronto, Oxford University Press.

Nadasdy, Paul, 2002. «Property and Aboriginal Land Claims in the Canadian Subarctic : Some Theoretical Considerations ", American Anthropologist, 104 (1) : 247261.

- 2003. Hunters and Bureaucrats. Power, knowledge and aboriginal-state relations in the southwest Yukon. Vancouver, UBC Press.

Nasr, Wren et Colin Scott, 2010. « The Politics of Indigenous Knowledge in Environmental Assessment : James Bay Crees and Hydroelectric Projects ». In P. Rethmann, I. Szeman et W. Coleman, Cultural Autonomy. Frictions and Connections, p.132-155. Vancouver, UBC Press.

Niezen, Ronald, 1993. "Power and Dignity : The Social Consequences of Hydro-Electric Development for the James Bay Cree ", Canadian Review of Sociology and Anthropology. La Revue Canadienne de Sociologie et d'Anthropologie, 30 (4) : 510-529.

- 1998. Defending the Land. Sovereignty and Forest Fife in James Bay Cree Society. Needham Heights, Allyn and Bacon.

Papillon, Martin, 2012. « Les peuples autochtones et la citoyenneté : quelques effets de contradictoires de la gouvernance néolibérale ", Éthique publique, 14 (1) : 2-15.

Pasternak, Shiri, 2013. On Jurisdiction and Settler Colonialism: The Algonquins of Barriere Lake Against the Federal Land Claims Policy, thèse de doctorat, département de géographie, University of Toronto.

Poirier, Sylvie, 2000. « Contemporanéités autochtones, territoires et (post)colonialisme : réflexions sur des exemples canadiens et australiens ", Anthropologie et Sociétés, 4 (1) : 137-153.

- 2001. Territories, Identity, and Modernity Among the Atikamekw (Haut St-Maurice, Quebec). In C. Scott, Aboriginal Autonomy and Development in Northern Quebec and Labrador, p. 98-116. Vancouver, UBC Press.

- 2004. "The Atikamekw : Reflections on their Changing World ». In B. Morrison et R. Wilson, Native Peoples : the Canadian Experience, p. 129-152. Oxford, Oxford University Press.

- 2008. "Reflections on Indigenous Cosmopolitics-Poetics ", Anthopologica, 50 (1) : 75-85.

- 2010. « Change, Resistance, Accommodation and Engagement in Indigenous Contexts : A Comparative (Canada-Australia) Perspective ", Anthropological Forum, 20 (1) : 41-60.

- 2013. « The Dynamic Reproduction of Hunter-Gatherers Ontologies and Values ». In M. Lambek et J. Boddy (dir.), A Companion to the Anthropology of Religion, p. 50-68. London, Wiley-Blackwell.
2017. « Nehirowisiw Territoriality : Negotiating and Managing Entanglement and Coexistence ». In Sylvie Poirier et Françoise Dussart (dir.), Entangled Territorialities. Negotiating Indigenous Lands in Australia and Canada, p. 212-134. Toronto, University of Toronto Press.

Poirier, Sylvie et Jean-Marc Niquay, 1999. Le Droit coutumier Atikamekw : pistes de réflexion. Rapport de recherche. Non publié.

Preston, Richard, 1986. «Introduction : Reflections on Territoriality », Anthropologica, 28 (1-2) : 11-17.

—. 2002 [1975]. Cree Narrative. Ottawa, National Museum of Man.

Preston, Susan, 2011. " Lifeworlds and Property : Epistemological Challenges to Cree Concepts of Land in the Twentieth Century ». In W. Coleman (dir.), Property, Territory, Globalization, p. 56- 79. Vancouver, UBC Press.

Pulla, Siomonn, 2003. " Franck Speck and the Moisie River Incident : Anthropological Advocacy and the Question of Aboriginal Fishing Rights in Quebec ", Anthropologica, 45 (1) : 129-145.

- 2006. Anthropological Advocacy? Frank Speck and the Mapping of Aboriginal Territoriality in Eastern Canada, 1900-1950. Thèse de doctorat, Departement of Sociology and Anthropology, Carleton University.

—. 2008. " "Would you believe that, Dr. Speck?" Frank Speck and The Redman's Appeal for Justice ", Ethnohistory, 55 (2) : 183-201.

—. 2011. «A Redirection in Neo-Evolutionism? A Retrospective Examination of the Algonquian Family Hunting Territories Debates ", Histories of Anthropology Annual, 7 : 170-190.

Richardson, Boyce, 1975. Strangers Devour the Land. Toronto, MacMillan.

Roark-Calnek, Susan, 1995. Algonquins of Barriere Lake Toponymy Project. State University of New York. Final Report (non publié).

—. 1996. Algonquins of Barriere Lake Social Customs Proiect: Indigenous knowledge Program. Algonquins of Barriere Lake Trilateral Secretariat. Final Report (non publié).

Salée, Danièle, et Carole Lévesque, 2010. « Representing Aboriginal Self-Government and First Nations / State Relations : Political Agency and the Management of the Boreal Forest in Eeyou Istschee ", International Journal of Canadian Studies, 41 : 99-135.

Salisbury, Richard F., 1986. A Homeland for the Cree. Regional Development in James Bay, 1971-1981. Montreal, McGill-Queen's University Press.

Samson, Colin, 2003. A Way of Life That Does Not Exist: Canada and the Extinguishment of the Innu. Londres, Verso.

Scott, Colin, 1982. " Production and Exchange among the Wemindji Cree : Egalitarian Ideology and Economic Base », Culture, 2 (3) : 51-64.

_. 1984. "Between "Original Affluence" and Consumer Affluence : Domestic Production and Guaranteed Income For James Bay Cree Hunters ", In R. Salisbury et E. Tooker (dir.), Affluence and Cultural Survival, p. 74-86. Washington, American Ethnological Society. 
1986. « Hunting Territories, Hunting Bosses and Communal Production among Coastal James Bay Cree », Anthropologica, 28 (1-2) : 163-173.

- 1989. « Knowledge Construction among Cree Hunters : Metaphors and Literal Understanding ", Journal de la Société des Américanistes, 75 : 193-208.

—_ 1997 [1988]. " Property Practice and Aboriginal Rights among Quebec Cree Hunters ». In T. Ingold, D. Riches et J. Woodburn (dir.), Hunters and Gatherers. Volume 2 : Property, Power and Ideology, p. 35-51. Oxford, Berg.

—. 2001. Aboriginal Autonomy and Development in Northern Quebec and Labrador. Vancouver, UBC Press.

- 2004. « Conflicting Discourses of Property, Governance and Development in the Indigenous North ». In Mario Blaser, Harvey Feit et Glenn McRae (dir.), In the Way of Development. Indigenous Peoples, Life Projects and Globalization, p. 299-312. New York, Zed Books.

— . 2013. « Le partage des ressources au Québec : perspectives et stratégies autochtones ». In A. Beaulieu, S. Gervais et M. Papillon (dir.), Les Autochtones et le Québec. Des premiers contacts au Plan Nord, p. 363-384. Montréal, Presses de l'Université de Montréal.

— . 2017. "The Endurance of Relational Ontology:

Encounters between Eeyouch and Sport Hunters ». In Françoise Dussart et Sylvie Poirier (dir.), Entangled Territorialities. Negotiating Indigenous Lands in Australia and Canada, p. 51-69. Toronto, University of Toronto Press.

Scott, Colin, et James Morrison, 2004. «Frontières et territoires : mode de tenure des terres des Cris de l'Est dans la région frontalière Québec/Ontario - I - Crise et effondrement ", Recherches amérindiennes au Québec, 34 (3) : 23-43.

—. 2005. "Frontières et territoires : Mode de tenure des terres des Cris de l'Est dans la région frontalière Québec/ Ontario - II - Reconstruction et renouveau ", Recherches amérindiennes au Québec, 35 (1) : 41-56.

Speck, Franck, 1915. « The Family Hunting Band as the Basis of Algonkian Social Organization ", American Anthropologist, 17 (2) : 289-305.

. 1923. "Mistassini Hunting Territories of the Labrador Peninsula », American Anthropologist, 25 : 452-471.
1927. « Family Hunting Territories of the Lake St. John and Neighbouring Bands ", ANTHROPOS: International Review of Anthropology and Linguistics, 22 : 387-403.

Speck Franck, and Loren Eisely, 1939. « Significance of Hunting Territory Systems of the Algonkian in Social Theory ", American Anthropologist, 41 (2) : 269-280.

Tanner, Adrian, 1971. " Existe-t-il des territoires de chasse ? ", Recherches amérindiennes au Québec, 1 (4-5) : 69-83.

- 1979. Bringing Home Animals. Religious Ideology and Mode of Production of the Mistassini Cree Hunters. London, C. Hurst.

- 1983. «Algonquian Land Tenure and State Structures in the North ", Canadian Journal of Native Studies, 3 (2) : $311-320$.

—. 1986. « The New Hunting Territory Debate : An Introduction to some Unresolved Issues ", Anthropologica, 28 (1-2) : 19-36.

Tsing, Anna, 2005. Friction. An Ethnography of Global Connections. Princeton, Princeton University Press.

Viau, Roland, 1995. « L'autopsie d'un contact : 1600-1900 ». In Odette Vincent (dir.), Histoire de l'Abitibi-Temiscamingue, p. 123-159. Québec, Institut Québécois de recherche sur la culture.

Whiteman, Gail, 1998. Tallymen talking: "On work on the land”. The Cree tallyman as an ecologically embedded business manager. (Presentation given at the Seventh Annual Conference of the International Association for the Study of Common Property, IASCP98, Vancouver, Canada, 10-14 June 1998).

- 2004. «The impact of Economic Development in James Bay, Canada ", Organization \& Environment, 17 (4) : 425448.

Wyatt, Stephen, 2004. Co-existence of Atikamekw and Industrial Forestry Paradigms. Occupation and Management of Forestlands in the St-Maurice River Basin, Québec. Thèse de doctorat, département de géomatique, Université Laval.

Wyatt, Stephen, et Yvon Chilton. 2014. « L'occupation contemporaine du Nitaskinan par les Nehirowisiwok de Wemotaci ", Recherches amérindiennes au Québec, 44 (1) : $61-72$. 